\title{
Transición entre lo instituido e instituyente en las teorías sexuales infantiles*
}

\author{
Transition between the Instituted and the Instituting in Child Sex Theories \\ Leidy Bibiana Camacho Ordóñez ${ }^{1}$
}

Para citar este artículo: Camacho, L. B. (2017). Transición entre lo instituido e instituyente en las teorías sexuales infantiles. Infancias Imágenes, 16(2), 257-271.

\section{Resumen}

Las perspectivas infantiles frente a los componentes de la sexualidad corresponden a un discurso plural y antagónico, alimentado por constructos sociales e históricos de carácter instituido, que tiende a perpetuar el predominio de lógicas tradicionales respecto al tema; y de carácter instituyente, pues contempla emergentes formas de percibir la identidad de género, rol de género y orientación sexual. Lo anterior es el resultado de un estudio realizado para comprender las teorías que han elaborado 12 niños y niñas de Buenos Aires, Argentina, entre los 5 y 6 años sobre la sexualidad, mediante el empleo de una metodología cualitativa, con técnicas lúdicas enmarcadas dentro del método clínico piagetiano. Estos resultados podrían constituirse en insumo para la consolidación de proyectos educativos en sexualidad desde temprana edad.

Palabras clave: teorías sexuales infantiles; identidad de género; rol de género; orientación sexual; infancia.
Recibido: 14-diciembre-2016 / Aprobado: 22-agosto-2017

\begin{abstract}
Child perspectives regarding sexuality components correspond to a plural and antagonistic speech, nourished by social and historical constructs of instituted nature which tends to perpetuate the prevalence of traditional logics with respect to the subject, and of an instituting character, which contemplates emerging forms of perceiving gender identity, gender role, and sexual orientation. This is the result of a study carried out to understand the theories that have developed 12 children from Buenos Aires, Argentina, between the ages of 5 and 6, on sexuality, by using a qualitative methodology, through the use of a qualitative methodology, with playful techniques framed Within the clinical method Piagetian. These results could constitute an input for the consolidation of educational projects in sexuality from an early age.
\end{abstract}

Keywords: child sex theories; gender identity; gender role; sexual orientation; childhood.

\footnotetext{
* El artículo es parte de la tesis La sexualidad desde la perspectiva infantil, con la que se obtuvo el título de Magíster en Problemáticas Sociales Infanto Juveniles de la Universidad de Buenos Aires, en mayo de 2014, con la tutoría del Dr. Horacio Esteban Belgich.

2 Psicóloga. Magíster en Problemáticas Sociales Infanto Juveniles, Universidad de Buenos Aires. Estudiante de Doctorado en Ciencias Sociales, Niñez y Juventud, Centro de Estudios Avanzados en Niñez y Juventud, Fundación Cinde, Universidad de Manizales. Docente, Universidad de Ibagué. Correo electrónico: leidy.camacho@unibague.edu.co
} 


\section{Introducción}

La sexualidad es un aspecto esencial de la condición humana, producto de una construcción histórico-social que comprende mucho más que el componente biológico (Aguirre et al., 2008; Belgich, 2004; Katchadourian, 2000; Marina, 2002; Ministerio de Educación Nacional de Colombia y Fondo de Población de las Naciones Unidas, 2008). Está estructurada por componentes medulares como la identidad de género, la orientación sexoerótica y el rol de género; y por las funciones reproductiva, erótica y comunicativa afectiva (González y Castellanos, 1996).

Por interés particular en este estudio, a partir del reconocimiento de la sexualidad infantil como dimensión humana y motor de constitución subjetiva de todo sujeto, se acoge el planteamiento freudiano (1908/1994) el cual sugiere que alrededor de los 3 y 5 años surge en los niños y niñas el interés de saber sobre el tema de la sexualidad y elaboran creencias al respecto, transformándolas en teorías sexuales infantiles. Sin embargo, cuando en esta investigación se usa el término teorías sexuales infantiles se hace en el sentido blando que se deduce de los trabajos de Piaget $(1976 ; 1991)$ y Freud (1905/1993; 1907/1996; 1908/1996), al respecto, para hacer referencia a las explicaciones que los niños y las niñas ofrecen acerca de cualquier tema, en este caso, de la sexualidad. Aquí se aclara que el foco de interés que se aborda en este artículo corresponde a los resultados obtenidos sobre la identidad de género, la orientación sexual y el rol de género, como componentes de la sexualidad, pero no de sus funciones. Estos componentes corresponden igualmente a las categorías de análisis del estudio.

Asimismo, en el marco de los resultados derivados de esta investigación, emergió la necesidad de retomar las categorías conceptuales de instituido e instituyente, propuestas por Castoriadis (1975), para alcanzar una lectura comprensiva de los hallazgos. En este sentido, por instituido se hace referencia a aquellas significaciones de los niños y niñas que al ser construidas en contextos histórico-sociales específicos están permeadas por ciertos discursos tradicionalistas de la sexualidad, reproductores de cierto orden social y humano. Así entonces, lo instituido corresponde a lo que ya está creado y que es impuesto al psiquismo durante el largo proceso de constitución subjetiva del sujeto. En relación con lo anterior, la categoría de instituyente o imaginario social radical representa aquellos elementos innovadores hallados en el discurso de los y las participantes en el estudio como reflejo de la creación y apertura hacia nuevos saberes sobre el tema y que rompen con aquellos mandatos sociales tradicionales que han acompañado históricamente a la sexualidad.

Por lo anterior, se plantea un interés por el caso particular de las teorías sexuales de los niños y niñas, en relación estrecha con su contexto cultural, social, escolar y familiar. Además, esto conlleva al abordaje de dos elementos centrales en el planteamiento del problema, relacionados con la información diversa y disociada que circula actualmente respecto al tema de la sexualidad.

\section{La situación en el contexto escolar}

El contexto escolar se constituye en una fuente de información importante para la elaboración de perspectivas infantiles alrededor de la identidad, el rol de género y la orientación sexual. Sin embargo, la realidad escolar del contexto argentino evidencia una resistencia actual en la implementación de la educación sexual desde temprana edad en las escuelas. Una muestra de ello corresponde al análisis que sobre las políticas públicas de la educación sexual en instituciones educativas, de la ciudad de Buenos Aires, realizaron Del Río Fortuna y Lavigne (2010), al plantear desde la década de 1980 hasta la actualidad el surgimiento de toda clase de resistencias y acciones penales frente a estas propuestas educativas.

En este sentido, aunque en la Ley 26.150 de 2006, Programa Nacional de Educación Sexual Integral, se plantea la obligatoriedad de brindar educación sexual integral a todos los educandos en los establecimientos educativos públicos, se registra al parecer una negativa ante el tema y cierta resistencia a su abordaje educativo. Así lo evidencian Antúnez, Durrieu y Kohen (2011), Barberis, Taborda y Zamanillo (2011), Boy (2011), Di Leo (2009), Ramos (2009) y Tomasini (2010), quienes, adicionalmente, sugieren la existencia en la escuela de un currículo oculto, que transmiten los y las docentes 
cotidianamente en su ejercicio profesional, relacionados con modos de ser para una sexualidad hegemónica, androcéntrica y heteronormativa.

\section{El papel de las actuales sociedades glo- balizadas y de consumo}

En el contexto social actual de grandes ciudades como Buenos Aires, vale la pena de igual forma reconocer la influencia que pueden ejercer la industria del entretenimiento, los medios masivos, la publicidad o las sociedades de consumo en la construcción de perspectivas infantiles de sexualidad. De acuerdo con Ayala, este constante "bombardeo" de información mediática, "no sólo insinúa, sino que muestra, expone, ofrece y vende nomenclaturas, modelos de ser y parecer; es más, expondría unos modos de sentir la vida (una intimidad prefabricada)" (2007, p. 27), que desvirtúa o empobrece el verdadero significado de la sexualidad. Por ejemplo, autores como Bernal (2006), Bonilla (2010), Lifschitz et al. (2008), Minzi (2006) y Urresti (2008) también comparten esta idea y puntualizan en la reproducción de nociones hegemónicas en las relaciones de género y la intolerancia hacia las diversas formas de identidad y orientación sexual, a través de las tandas de comerciales dirigidas al público infantil, el cine infantil, juguetes, anuncios publicitarios, entre otros.

Esta influencia mediática en la constitución subjetiva de la sexualidad, de acuerdo con Carli (septiembre 18 de 2011), ha llevado a que los niños y niñas sean hoy vistos más como consumidores que como escolares, tironeados por la escuela, por la publicidad, por el mercado y por los mass media en simultáneo. Teóricos como Baquero, Diker y Frigerio (2007), Curia (2006), Martín-Barbero (2002), Ortiz (1997; 2004), Steinberg y Kincheloe (1997) y Tenti (2007) señalan igualmente esta instalación del mercado y los medios de comunicación masiva como nuevos referentes de legitimación cultural.

Lo anterior plantea la necesidad de reconocer el multidireccional y complejo proceso de socialización infantil actual y, por tanto, de comprender la construcción de perspectivas infantiles de sexualidad, en relación estrecha con el contexto escolar, familiar, religioso, político y mediático. Sin embargo, en el ámbito académico poco ha sido el interés por profundizar en este tema desde el punto de vista infantil. En los estados del arte construidos por Citeli (2005), Gogna (2005) y Valdés y Guajardo (2007) se aprecia un amplio interés del mundo académico latinoamericano por abordar temas como la maternidad temprana, el VIH-sida y sus formas de prevención, el género, la educación sexual y las políticas públicas en sexualidad, con la participación de jóvenes y adultos, no con niños y niñas.

Durán y Rojas (1996) también coinciden en señalar la realización de múltiples estudios sobre el tema con población adolescente y concluyen que los escasos estudios que exploran el punto de vista infantil asumen un enfoque médico del riesgo y la prevención de la violencia sexual hacia la infancia y la niñez. No obstante, vale la pena aquí mencionar las investigaciones de Bachs (1984), Ballester y Gil (2006), Bonilla (2010), Camacho y Trujillo (2009), Sverdlik (1996) y Vanegas, Bonilla y Camacho (2010) que, al aproximarse al estudio de la sexualidad desde la perspectiva de los niños y niñas, se constituyen en referentes empíricos importantes en el marco del estudio que aquí se presenta.

Por lo descrito anteriormente, este estudio plantea como objetivo comprender las teorías que se han construido sobre la sexualidad en un grupo de niños y niñas argentinos de 5 y 6 años, de la ciudad de Buenos Aires. Al respecto, y como se aclaró al inicio de este apartado, para efectos de publicación en este artículo se retoman solamente los hallazgos obtenidos en las categorías identidad de género, rol de género y orientación sexual. Es por ello por lo que una de las preguntas planteadas en esta investigación, y sobre la cual se focaliza este artículo, es: ¿cuáles son las teorías sexuales construidas por un grupo de niños y niñas argentinos de 5 y 6 años, de la ciudad de Buenos Aires, acerca de la identidad de género, la orientación sexual y el rol de género, como componentes fundamentales de la sexualidad?

\section{Método}

Enfoque y diseño de investigación

Esta es una investigación de enfoque cualitativo, ya que pretende comprender las teorías que en relación con los componentes de la sexualidad 
han construido un grupo de niños y niñas argentinos (Camacho, 2015). Este tipo de enfoque elegido, según Sandoval (2002), agrupa una serie de metodologías que facilitan la comprensión de la realidad social desde las interpretaciones asignadas por sus protagonistas, desde sus contextos particulares y reivindicando elementos subjetivos e intersubjetivos.

En el diseño de investigación se adoptó el método clínico de Piaget como recurso para la comprensión del pensamiento infantil, el cual consiste en conversaciones abiertas entre el investigador y los niños y las niñas mientras explican la solución de algún problema, realizan alguna tarea o participan en un juego. Consiste en un método abierto y flexible que, de acuerdo con Delval (2001), no está necesariamente ligado a la teoría piagetiana y los procedimientos pueden adaptarse o ajustarse según el problema que se quiere estudiar. Se aclara que, en este estudio, se dista del interés de esta metodología de generalizar las concepciones encontradas en el grupo participantes y trasciende la mirada evolutiva y longitudinal de la lógica del pensamiento infantil.

Asimismo, un elemento esencial de este método corresponde a la construcción de hipótesis de partida que, de acuerdo con Delval (2001), permiten sugerir expectativas sobre la forma en que los participantes van a abordar el objeto de estudio. Al respecto, resulta fundamental aclarar que, en el marco de este estudio, estas hipótesis de carácter cualitativo no son empleadas con el objetivo de verificarlas, sino que funcionan como guía para orientar el proceso de diálogo con los niños y las niñas, y no como suposiciones previas o como afirmaciones a verificar. Se trata más bien de hipótesis plausibles, o como diría Martínez (2006) "provisionales, y se van modificando durante el proceso, para no estrechar nuestra perspectiva y visión de la realidad" (p. 132).

En este sentido, las siguientes hipótesis se construyeron desde los resultados de los pocos estudios encontrados sobre el tema de la sexualidad desde la perspectiva infantil (Bachs, 1984; Sverdlik, 1996; Camacho y Trujillo, 2009; Vanegas, Bonilla y Camacho, 2010), y por cada componente de la sexualidad:
1. Los niños y las niñas construyen teorías sobre su identidad de género a partir de la identificación con la figura materna y/o paterna y acuden tanto a elementos de tipo biológico como cultural para explicarla.

2. Los niños y las niñas definen su orientación sexual a partir de la identificación con las figuras masculinas o femeninas y las interacciones que estas figuras establecen entre ellos y por sus pulsiones sexuales. En este proceso construyen una aceptación cultural hacia las relaciones humanas heterosexuales e intolerancia hacia la homosexualidad.

3. Los niños y las niñas explican el rol de género a partir de la adscripción naturalizada que hacen de los hombres y las mujeres a determinadas prácticas domésticas, profesionales, laborales y lúdicas. De igual forma, no poseen concepciones que reflejen un avance hacia el reconocimiento de la equidad en las relaciones de género.

Con los resultados derivados del estudio se evidencia cómo estas hipótesis, que funcionaron como guía de trabajo, son susceptibles de cambio a medida que emergen y se revelan nuevos datos durante la interacción con los y las participantes y el posterior análisis de la información.

\section{Procedimiento}

El estudio se desarrolló teniendo en cuenta los siguientes momentos investigativos, que favorecen el ahondamiento progresivo del saber en torno al objeto de estudio (González, 2000; Sandoval, 2002; Quintana, 2006), sin que implique un proceso rígido, lineal o independiente en el desarrollo de cada una de las fases.

\section{Momento exploratorio}

Corresponde a la fase de establecimiento de contactos con autoridades competentes, las instituciones educativas y la selección de los y las participantes. De esta forma, y como parte de las condiciones éticas del estudio, se tramitó inicialmente el consentimiento informado con la Dirección General de Planeamiento Educativo, la Dirección Educativa de Educación Primaria y la Dirección Educativa de 
Educación Inicial, del Ministerio de Educación del Gobierno de la ciudad de Buenos Aires, quienes después de evaluar el estudio asignaron el aval por cumplir con los requisitos formales establecidos en la Resolución núm. 3.906/SED/04. Posteriormente, se obtuvo la autorización por parte de la Supervisión de Educación Inicial del Distrito Escolar N. ${ }^{\circ}$ 1, la Supervisión de Educación Primaria del Distrito Escolar N. ${ }^{\circ}$ 1, las instituciones educativas, los padres y madres de familia de los niños y niñas seleccionados, así como el asentimiento verbal y voluntario de los y las participantes.

Finalmente, la unidad de trabajo estuvo constituida por seis niños y seis niñas, argentinos, con edades entre los 5 y 6 años y pertenecientes a dos instituciones educativas estatales de la ciudad de Buenos Aires (Argentina). Los criterios de selección de esta población fueron intencionales: niños y niñas escolarizados en instituciones públicas, con la edad requerida en el estudio (por ser un período importante para la construcción de teorías sexuales y un rango de edad escasamente abordado a nivel investigativo). Adicionalmente, desde la orientación de los directivos y las maestras de los niños y niñas, se seleccionaron aquellos que asisten regularmente a la escuela, pertenecen a estratos socioeconómicos bajos, presentan facilidad de expresión y, por supuesto, que manifestaron su participación voluntaria y cuyos padres y madres de familia asignaron su consentimiento verbal y escrito.

\section{Momento descriptivo}

Constituye el trabajo de campo o de indagación de las perspectivas infantiles sobre los componentes de la sexualidad. Para ello, se diseñaron seis talleres, validados previamente con la aplicación de prueba piloto, que se desarrollaron en 31 sesiones con una duración aproximada de 40 minutos por encuentro. En el caso del jardín de infantes, se desarrollaron 15 sesiones en un salón de clases; y 16 encuentros en el caso de la escuela primaria, en diferentes días y horarios escolares. En cuanto al número de participantes por sesión, se menciona que algunos talleres se realizaron con 6 niños y niñas, otros con tres personas o por parejas, dependiendo de la técnica.
Cada taller plantea en su estructura un objetivo central y una técnica de recolección lúdica específica. Esto con el fin de promover la interacción dialógica y espontánea entre los niños y niñas y la investigadora. Del mismo modo, para responder a los criterios del método clínico piagetiano, ante cada actividad o juego propuesto se espera la emergencia de comentarios infantiles espontáneos. Si estos no se producen, desde preguntas neutras planteadas por la investigadora, se buscaba estimular la expresión en los y las participantes.

El primer taller se orienta a propiciar un ambiente lúdico inicial para el reconocimiento mutuo y la comunicación espontánea, entre la investigadora, niñas y niños. En el segundo encuentro los participantes construyen y narran una historieta a partir de la organización de láminas proyectivas que contienen imágenes alusivas al tema del estudio; estas láminas fueron diseñadas en el marco de la investigación para desencadenar las narraciones. La tercera de las técnicas permite a los sujetos el uso de juguetes que aluden objetos (sartén, auto, pulsera, bolsa, máquina excavadora, avión, balón de fútbol, billetes, peineta color rosado), esto con el fin de indagar acerca de la identidad y el rol de género, mientras se dialoga sobre el uso de estos objetos.

La técnica del dibujo consiste en concursar para lograr el dibujo más parecido a dos muñecos sexuados desvestidos (hombre y mujer) que se exponen en una mesa, mientras se habla sobre la identidad de género. Asimismo, en otro taller se induce a que los niños y niñas propongan desvestir y vestir a una pareja de muñecos, hombre y mujer, con ropa de modo contrario a lo señalado por la cultura de género, mientras se conversa con ellos y ellas sobre la orientación sexual, identidad y rol de género. En el último taller se propone jugar con un material didáctico denominado Lotería Equisex, que contiene imágenes y situaciones relacionadas con los componentes de la sexualidad.

\section{Momento interpretativo}

Esta fase final se relaciona con el análisis de los datos. En este sentido, los resultados que se describen a continuación se procesaron tomando los aportes de la teoría fundamentada de Strauss y Corbin (2002), como una metodología cualitativa que 
permite aproximarnos a la comprensión de fenómenos, empleando con fidelidad lo expresado por los informantes.

Aunque uno de los objetivos primordiales de esta metodología es construir teoría, es importante aclarar que en este estudio la intención no es esa. Por el contrario, se asume el método como un recurso orientador en el proceso de análisis sistemático de los datos que es útil para construir una interpretación comprensiva de las perspectivas infantiles de los y las participantes. Es decir, que desde el eje o categoría central titulada "transición o interjuego entre lo instituido e instituyente en las perspectivas infantiles de los componentes de la sexualidad y la necesidad de acompañamiento educativo", que emergió con el análisis de los datos, se acude en la etapa final de análisis a diversos referentes teóricos ya creados por otros autores para intentar comprender los hallazgos. Por tanto, no se construye una teoría propia del estudio a partir de los datos recolectados.

Del mismo modo, para el logro de tal propósito, al momento de analizar la información esta investigación retoma las tres fases de la propuesta de Strauss y Corbin (2002) y que serán descritas y desarrolladas en el apartado de los resultados: codificación abierta o descripción, codificación axial o interpretación y codificación selectiva o teorización.

\section{Ética del estudio}

Se tuvo en cuenta las condiciones académicas, técnicas y administrativas de la Resolución n. ${ }^{\circ}$ 3.906/ SED/04, para la realización de estudios en unidades educativas de gestión estatal, dependientes de la Secretaría de Educación de la Ciudad Autónoma de Buenos Aires. Como se mencionó anteriormente, la viabilidad y las condiciones éticas del estudio fueron evaluadas por el Ministerio de Educación del Gobierno de la ciudad de Buenos Aires, y se obtuvo el consentimiento informado de las instituciones educativas, los padres y madres de familia y los niños y niñas seleccionados.

\section{Validez y confiabilidad}

El estudio cumple con el criterio de la coherencia o consistencia lógica que se soporta en la integración de la postura teórica de la investigadora y el diseño metodológico seleccionado (Hernández, Fernández y Baptista, 2007). Del mismo modo, adopta la triangulación de teorías y la saturación de información por categorías. La descripción detallada y rigurosa del proceso empleado respalda la credibilidad de la investigación.

\section{Resultados}

\section{Codificación abierta: una aproximación descripti-} va a las voces de los niños y las niñas

En esta fase, los datos obtenidos se descomponen en partes discretas y se comparan en busca de similitudes y diferencias. De este modo, empiezan a emerger códigos que se asignan a determinados datos considerados significativos y se agrupan en las categorías definidas en el estudio.

La tabla 1 presenta los códigos que emergieron dentro de cada una de las categorías de análisis del estudio, durante esta primera fase de análisis.

Tabla 1. Fase de codificación abierta o descripción del análisis de los datos.

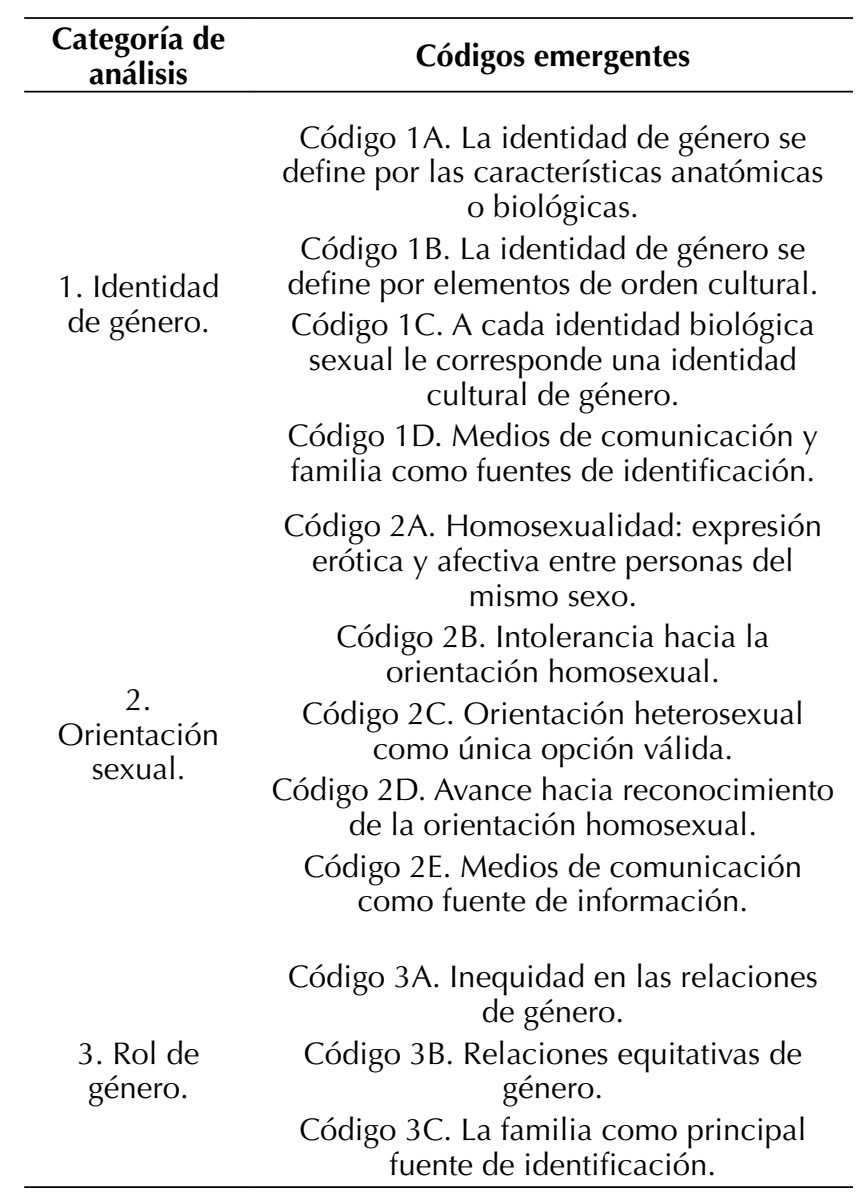


En esta fase de análisis, conviene recordar que el objetivo está orientado a visibilizar de manera descriptiva los relatos de los niños y niñas participantes en el estudio; relatos que fueron organizados dentro de las categorías definidas (componentes de la sexualidad) y en una serie de 12 códigos identificados por la investigadora tras la lectura minuciosa de las transcripciones de los talleres realizados. Este primer proceso de análisis de los datos se constituye en base fundamental para avanzar en el nivel interpretativo de la información en las dos etapas siguientes de la teoría fundamentada. De este modo, como se trata de un proceso de análisis circular y dinámico, a continuación, se presentan los hallazgos obtenidos en la fase de codificación axial, que articula, asimismo, los relatos más relevantes de los niños y niñas derivados de la primera etapa.

\section{Codificación axial o interpretación: la emergencia} de las teorías sexuales infantiles

En esta segunda etapa de análisis comparativo constante se realiza una reagrupación de los datos que se fracturaron durante la codificación abierta para formar explicaciones más precisas y completas. Se identifican así las coincidencias de sentido entre las categorías, para darle paso a la conformación de nuevas categorías, y que corresponden a las teorías sexuales infantiles, en el caso de este estudio.

Se reitera que el concepto de teoría se asume aquí no con una pretensión universalista, sino para hacer referencia a los discursos que los niños y niñas participantes expresan respecto a cada uno de los componentes de la sexualidad, reflejo de sus formas particulares de comprender el mundo; como un sujeto que es influido por las significaciones culturales de sus contextos, pero también, desde su lugar como sujeto activo, agente y creador de nuevas miradas sobre su realidad social.

En cada una de las teorías encontradas durante la segunda etapa se citan algunos relatos expresados

\footnotetext{
5 Los relatos de las niñas y los niños se presentan entre comillas y se aclara entre paréntesis el autor de las expresiones, para identificar el género y la edad, así: (Niño/5), (Niña/6), y la letra (I) que representa la intervención de la investigadora. Entre paréntesis y en cursiva también se aclara el objeto o situación hacia el cual los niños, niñas o la investigadora orientan el diálogo; Por ejemplo: (ficha de lotería-hombre y mujer), (muñeco sexuado).
}

por los niños y las niñas en la fase anterior, como soporte comprensivo de estas teorías a la luz de las voces infantiles ${ }^{2}$. Del mismo modo, no con la intensión de verificar, se hace énfasis en cómo las teorías encontradas revelan nuevos datos en comparación con las hipótesis cualitativas iniciales planteadas en este estudio.

En este sentido, desde los códigos 1A, 1B, 1C, 1D de la tabla 1, y el establecimiento de relaciones entre los mismos, se enuncia la primera teoría o perspectiva infantil de sexualidad, dentro de la categoría identidad de género: el cuerpo y sus adornos como indicadores de identidad genérica. Esta perspectiva infantil condensa el conjunto de relatos emitidos por los y las participantes, que sugieren la relevancia no solo de ciertas características anatómicas o físicas en la definición de la identidad de género, sino también de ciertos rasgos asociados a construcciones de tipo cultural, como el uso de colores, prendas de vestir, accesorios o formas de lucir el pelo.

(I: ¿y qué diferencia hay en los cuerpos de estos dos? [Ficha de lotería-hombre-mujer]). Que uno tiene pito y la otra tiene pichi, el otro tiene tetillas y la otra tiene tetas ( $\mathrm{Niña} / 6)$.

Es nena. (I: ¿y por qué es nena?). Porque tiene color de nena, rosa, violeta (Niña/6). (I: ah, y yo no sabía eso, ¿quién les dijo a ustedes que el rosado era de las nenas?). Nadie, nosotros lo sabemos ( $\mathrm{Niño} / 5)$.

En el conjunto numeroso de relatos encontrados (los citados son solo una muestra) se aprecia el predominio de elementos de indumentaria a asociados a cada género, como indicador de la relevancia que tiene la cultura en el proceso comprensión y construcción de identidades de género.

Esta perspectiva infantil se puede corroborar con mayor fuerza con la segunda de las teorías encontradas dentro de esta categoría: el género masculino o femenino como únicas opciones válidas de identificación. De esta forma, se puede nacer hombre o mujer con rasgos anatómicos y características primarias y secundarias específicas para cada sexo, a los cuales se les asigna de manera directa una serie de accesorios culturales, que en su conjunto darán complemento a dos únicas formas de pensarse, 
sentirse y autopercibirse: una identidad femenina o una identidad masculina. Así, no existe espacio para dar cabida a otras posibilidades identitarias.

¡Horrible, horrible! (gritando) (Niño/5). Un varón no se puede vestir con ropa de nenas y las nenas no se pueden vestir con ropa de varón (Niña/5).

Él es gay porque se viste así. (I: ¿y a ustedes que les parece estas personas que visten así?). Son asque$\operatorname{rosos}(\mathrm{Niña} / 6)$.

De ahí el notorio rechazo moral encontrado en las narrativas infantiles hacia el uso de elementos estéticos contrarios a lo que establece la cultura. De esta manera, la no coincidencia entre la identidad biológica sexual y la identidad cultural de género construida para ese sexo biológico específico despierta en los niños y niñas reacciones de burla, risa, desagrado, asco, vergüenza, intolerancia y agresión. En palabras de Zanbrini, se diría "que en la matriz heterosexual las identidades genéricas deberán ser coherentes con los cuerpos sexuados para hacer posible la inteligibilidad y la regulación de los cuerpos" (2008, p. 126).

Con respecto de las fuentes identificatorias relevantes, se encuentra que los padres proporcionan elementos de identidad que profundizan el reconocimiento exclusivo del binomio mujer-hombre. De otro lado, aunque los medios de comunicación, la publicidad y la cotidianidad del contexto argentino ofrecen un panorama diverso de asumir identidades, las que no encajan en el modelo hegemónico de identidad están siendo censuradas, a pesar de los últimos avances en ese país en materia legislativa, como es el caso de la Ley 26.743 de identidad de género. Esto, en relación con la hipótesis inicialmente planteada sobre la identidad de género, Ileva a reconocer que los referentes principales de identificación suelen ser los padres y madres de los y las participantes, aunque no de manera exclusiva, pues se articulan a este ejercicio constitutivo de la identidad otros modelos presentes en la cotidianidad del contexto argentino, los medios de comunicación, la publicidad y la internet.

Por otro lado, desde los códigos 2A, 2B, 2C, 2D y $2 \mathrm{E}$ emerge la teoría de la hegemonía heterosexual e intolerancia hacia la homosexualidad, que lleva a percibir la homosexualidad con desagrado, al asociarse con numerosas expresiones infantiles como: ¡qué asco!, iguácala!, ¡son asquerosos!; o también como objeto de burla y gracia: ¡son tontos!, ¡era gracioso!

(I: ¿saben qué?, este muñeco no tiene novia, sino que tiene un novio). ¡Son gayyyy! (grita) (Niña/6). (l: pero ¿qué les parece que él tenga un novio?). No me parece, son payasadas (Niño/6). ¡Ay es horrible! (Niña/6).

(I: ¿él se puede casar con otro señor?). No, es la ley (Niña/6). Sí, no se permite, se pueden casar, pero hombres y mujeres (Niño/6). (I: ¿y él puede tener un bebé con otro señor?). No porque es la ley también y no le pueden hacer a otro nombre las cosas (Niña/6). Sí, la ley dice que no (Niño/6). (I: iy si ellos no le hacen caso a la ley?). Lo arrestan, van a la cárcel (Niño/6). (I: ¿y ella con otra mujer pueden tener un bebé también?). Sí, por supuesto, sí, sí pueden, pero la ley no les deja (Niño/6).

El último relato pone en evidencia cómo la unión matrimonial y la tenencia de hijos en el marco de relaciones lésbicas o gay son vistas como una situación de transgresión a la norma que amerita rechazo o sanción penal. Paralelo a estas apreciaciones, los niños y niñas argumentan la validez exclusiva hacia la orientación hegemónica heterosexual y, por tanto, esta opera como referente privilegiado de identificación infantil, tal como se plantea en la segunda hipótesis cualitativa de este estudio.

En el marco del credo de las religiones judía y cristiana, encontramos una serie de códigos morales que guardan relación con estos planteamientos infantiles respecto la orientación sexual. Desde esta perspectiva, se considera a la sexualidad como natural y normal cuando gobierna la meta de la procreación y, por tanto, censura moralmente a la homosexualidad al oponerse a la finalidad procreadora del ser humano.

Lo anterior traza el camino hacia la comprensión integradora de los resultados que se vienen esbozando y que en términos generales plantea la coexistencia de elementos instituidos e instituyentes en las perspectivas infantiles de sexualidad. De este modo, es la categoría de lo instituyente la que 
remite a plantear la cuarta de las teorías identificadas en este estudio: teoría del respeto hacia la orientación sexual no convencional, que constituye un elemento innovador respecto a la hipótesis inicial construida en esta investigación. Esta teoría emerge de la apreciación de una de las niñas de 6 años participantes, que da cuenta de un leve pero significativo avance en el reconocimiento del matrimonio entre personas del mismo sexo:

(I: ¿si él [muñeco sexuado] tuviera un novio, se puede casar?). Sí. (I: ¿un hombre se puede casar con un hombre aquí en Argentina?). Sí, pasa muchas veces; también ya pasó por la tele, que un gay se casó con otro gay. (I: ¿y qué te parece eso?). Que está, también puede ser bien. (I: ¿te gustaría que los hombres se casaran entre hombres?). No, a mí no. (I: ah, pero tú habías dicho que te parecía bien). Un poquito (Niña/6).

Esto podría constituir, de manera hipotética, un importante resultado del eco que empieza a erigirse con fuerza desde que el 15 de julio de 2010 Argentina aprueba legalmente el matrimonio igualitario y la posibilidad de adopción dentro de estas relaciones, a través de la Ley 26.618 y el Decreto $1054 / 10$. Es por ello que, en medio de los numerosos testimonios infantiles de intolerancia hacia las orientaciones sexuales de tipo no convencional, resulta esperanzador encontrar al menos una opinión que se aproxima al reconocimiento de la igualdad humana.

Por otro lado, en la categoría rol de género se encuentran dos nuevas teorías infantiles. La primera de ellas se denomina la teoría de la inequidad de género, enraizada en visiones tradicionalistas de las relaciones de género tienden a través de la historia a asignar una serie de patrones de comportamiento específicos para cada sexo y a perpetuar relaciones soportadas en la inequidad en las relaciones entre hombres y mujeres.

(I: Pero tú ahora dijiste que tu mamá era más inteligente, ¿quién es más inteligente entonces?). Mi papá, pero cada cosa es en algo. (I: ¿o sea que tu mamá es inteligente en qué cosas?). En cosas de cocinar, hacer cosas, hacer pasteles (Niño/6).
(I: ¿las mujeres juegan fútbol?). No, el fútbol es para los hombres porque las nenas no tienen fuerza, el partido se juega con fuerzas (Niño/5).

Desde varios relatos infantiles encontrados se resalta la solidez de planteamientos con respecto de la correspondencia del ámbito privado al género femenino y del ámbito público al género masculino. En oposición a esta perspectiva infantil, y que asigna una mirada renovadora de la hipótesis cualitativa planteada en este estudio, aparece la teoría del reconocimiento de la igualdad en las relaciones género, la cual sugiere que tanto hombres como mujeres reúnen una serie de competencias para participar en actividades que culturalmente han sido asignadas al género contrario. Es importante aclarar que en cada uno de los niños y niñas se aprecia la coexistencia de ambas teorías, por lo que resulta común encontrar posiciones contradictorias que oscilan entre la equidad e inequidad. "A veces, mi papá es el más más vago que no plancha nunca, mi papá obliga a mi mamá a planchar, es revago" (Niño/6).

Dentro de esta categoría, planteamientos construidos a lo largo de la historia de la humanidad parecieran ejercer eco en las apreciaciones infantiles encontradas. Foucault (1976/2008), por ejemplo, sugiere la creencia de una condición de ternura natural específica de la mujer para ocuparse de los hijos y del hogar. Asimismo, Badinter (1981), menciona cómo en el marco del credo de la religión cristiana se corrobora una condición natural femenina ligada a la pasividad, sumisión o alienación y una superioridad del hombre. Todo el legado de tradiciones históricas humanas parece aún persistir en los imaginarios sociales. Sin embargo, junto a ellos han aparecido nuevas construcciones de sentido que permiten comprender de diferentes maneras la realidad. Ejemplo de ello ha sido el avance hacia el reconocimiento de la equidad entre los géneros.

Las seis perspectivas infantiles encontradas en los componentes de la sexualidad son indicadoras de la relevancia que tiene la cultura y el contexto en el proceso comprensión y construcción de identidades de género, orientación sexual y rol de género. Encontramos un abanico de prácticas, identidades y relaciones erótico-afectivas que van 
más allá de una heteronormatividad, pero que reciben una connotación negativa por parte de los niños y niñas vinculados al estudio.

De acuerdo con Werner (2008), existe un proceso de reproducción social generado por instituciones sociales como la familia, la escuela y los medios de comunicación, entre otros, que reproduce de manera naturalizada la homofobia, el heterosexismo y formas ortodoxas en las concepciones de género. Foucault (1974/2007; 1976/2008), explica una racionalidad moderna alimentada por una lógica de la identidad binaria que, siguiendo a Zanbrini (2008), fue la que sostuvo "una relación natural entre el sexo y el cuerpo, fortificando la matriz heterosexual como norma social hegemónica" (p. 127).

Con lo anterior, lo que se intenta aquí plantear es cómo las diversas maneras en que la sexualidad se vive, concibe y expresa, obedecen a configuraciones históricas que impone la cultura y que aportan un entramado discursivo diverso, complejo, dinámico, contradictorio y cambiante. No obstan-

te, se invita también al necesario reconocimiento del niño y niña como sujeto activo, constructor y partícipe de nuevas realidades.

\section{Discusión}

Al haber abordado las fases de categorización descriptiva y axial dentro de la propuesta de Strauss y Corbin (2002), desde la fase de codificación selectiva o teórica se avanza ahora hacia la búsqueda de un sentido integrador de los hallazgos hasta aquí presentados. De esta manera, se plantea la siguiente categoría central del estudio y se retoman, en seguida, diversos elementos teóricos y autores que permitan comprender las perspectivas infantiles sobre la identidad de género, orientación sexual y rol de género: transición o interjuego entre lo instituido e instituyente en las perspectivas infantiles de los componentes de la sexualidad y la necesidad de acompañamiento educativo.

La sexualidad, como construcción social e histórica y de carácter diverso, dinámico y complejo, lleva a comprender las construcciones de sentido contradictorias que han tejido niños y niñas alrededor de este tema y la identificación de elementos que transitan entre lo instituido e instituyente, lo tradicional o innovador.
Lo instituido en las perspectivas infantiles

Si nos centramos inicialmente en lo tradicional, las perspectivas infantiles apuntan al establecimiento de una correspondencia directa e invariante entre el cuerpo biológico hombre-mujer y un conjunto de características culturales específicas que excluye a los sujetos en devenir o que no se sienten identificados con los modelos hegemónicos de identidad. Esta mirada excluyente de la identidad sexual también aplica en lo concerniente a la orientación sexual no convencional, frente a la cual los niños y niñas han resultado moralmente descalificadores. De este modo, la heteronormatividad extiende su dominio en el plano de los roles de género, la cual opera como soporte de relaciones inequitativas entre mujeres y hombres.

Es así como estos planteamientos infantiles evidencian, entre otras cosas, la influencia de la ideología judeocristiana que nutre a modelos históricos y naturalizados de identidad, orientación sexual y rol de género. Lógicas que se inscriben en un contexto actual argentino globalizante y que ofrece a chicos y chicas múltiples referencias culturales para su constitución subjetiva de la sexualidad. Desde el entorno escolar, por ejemplo, autores como Antúnez, Durrieu y Kohen (2011), Barberis, Taborda y Zamanillo (2011), Boy (2011), Di Leo (2009), Ramos (2009) y Tomasini (2010) ponen en evidencia desde un contexto argentino el rol que desempeña la escuela actual en la transmisión de modelos hegemónicos de sexualidad.

Esta producción simbólica, desde la perspectiva de Lamas (2000) "contribuye ideológicamente a la esencialización de la feminidad y de la masculinidad" (p. 344) y a preponderar los valores masculinos sobre los femeninos (Cucchiari, 2000; Tomasini, 2010). Así entonces, es necesario pensar la lógica de género como una lógica de poder (Hall, 1997) y de dominación, que a manera de discursos sociales ya establecidos tienden a naturalizar, dominar o encauzar la vida social y humana (Belgich, 2004; Foucault, 1977/2006).

De este modo, en el marco de la sexualidad, ser hombre o mujer, con orientación exclusiva heterosexual, asumiendo roles o del ámbito público o del privado, correspondería a situaciones originadas desde discursos sociales e históricos suscritos 
bajo lógicas de poder. Esta cuestión de lo ya establecido remite entonces a pensar en la categoría de lo instituido. Desde el libro La institución imaginaria de la sociedad, Castoriadis (1975) inicia el estudio de la creación humana, del factor imaginario o el imaginario social. En esta obra, y reflexiones siguientes a ella, es posible comprender lo instituido o imaginario social efectivo, como aquel conjunto de significaciones construidas en contextos histórico-sociales específicos que operan como dispositivos o instituciones reproductoras de un orden social y humano con la intención de perpetuarse. En consecuencia, cada sujeto que nace es creado, fabricado, moldeado y humanizado en esta masa de significaciones históricas instituidas.

En este sentido, las doctrinas tradicionalistas persistentes aún en las perspectivas infantiles de sexualidad se comportan como verdaderas instituciones que han implicado el desarrollo de un proceso histórico compartido y que posibilitan, por ejemplo, que los cuerpos, identidades, roles y orientación sexual sean vistos como un hecho natural y no como una construcción histórico social.

La emergencia de lo instituyente: valiosa oportunidad de educación para la sexualidad desde temprana edad

Desde el planteamiento de los elementos instituidos, resulta relevante comprender que, aunque dichas instituciones históricas han reclamado cierta permanencia, han variado en el tiempo a partir de los elementos innovadores que las sociedades han incorporado a las antiguas doctrinas y que corresponde, en el caso de este estudio, a las perspectivas infantiles que reconocen la equidad en las relaciones de género y la tolerancia y aceptación de la orientación homosexual. De esta manera, y volviendo a Castoriadis, estos enunciados infantiles operarían como elementos instituyentes o imaginario social radical, como creación de nuevos saberes que introducen las sociedades con el paso del tiempo, que se edifica sobre las ruinas de los edificios simbólicos precedentes y que requiere, de acuerdo con Casati (2008), interpelar las teorías tradicionales.

De esta manera, los niños y niñas del estudio están elaborando nuevos marcos interpretativos que buscan acomodarse o encajar con otras visiones tradicionalistas de sexualidad. De este proceso, es decir, del interjuego entre lo instituido-instituyente, puede dar cuenta la contradicción entre la variedad de teorías halladas en este estudio. Asimismo, la tendencia prioritaria a comprender los componentes de la sexualidad desde imaginarios sociales instituidos es muestra de su solidez y dominio en la memoria colectiva e histórica del grupo social al que pertenecen los y las participantes.

De esta forma, se rescata el importante papel que empiezan a desempeñar estas nuevas construcciones de sentido sobre el tema de la sexualidad. Esto, sin la pretensión de establecer relaciones de causalidad, podría ser producto de una serie de cambios a nivel normativo, social, político, económico y mediático, registrados en las últimas décadas en el mundo y que hacen eco en el contexto argentino desde procesos de globalización o mundialización de la cultura (Ortiz, 2004). Como muestra de estos avances, se resaltan como relevantes las ya mencionadas Ley de Matrimonio Igualitario y la Ley de Identidad de Género.

Todos estos éxitos cosechados desde lo político, académico y normativo empiezan a ser metabolizados y reclaman un mayor espacio dentro de los imaginarios sociales. Al respecto, debe existir un mayor compromiso con la educación que dé lugar a la comprensión de las formas instituyentes de comprender y vivir la sexualidad humana, apuntando hacia la formación de sujetos democráticos, pluralistas y respetuosos de los derechos de los otros y las otras, y comprometidos con una educación sexual del diálogo franco y sincero. La coexistencia de teorías tradicionalistas e innovadoras hallada en la perspectiva de los niños y niñas participantes da cuenta de un proceso transitorio, que invita y convoca a la habilitación de espacios dialógicos y educativos con ellos y ellas sobre el tema de la sexualidad y aprovechar su capacidad crítica y reflexiva.

En este sentido, se considera que esta oportunidad de cambio y transformación de los imaginarios sociales se posibilita si se rescata la posición activa del sujeto como constructor de la realidad social y de cómo interpreta, resignifica y asume o no los discursos social e históricamente construidos. Este 
intento de reconocer el aspecto volitivo y reflexivo del sujeto en el proceso de constitución subjetiva se constituye en un valioso aporte que se puede extraer de las apreciaciones de Butler (2000) y Hall (1997). También Conway, Bourque y Scott (2000), por ejemplo, refirieren que:

[...] las instituciones no siempre tienen éxito en su tarea de inculcar conductas culturalmente aceptables o comportamientos convencionales. No parece que los individuos simplemente acepten o reflejen las designaciones normativas. Más bien, las ideas que tienen acerca de su propia identidad de género y su sexualidad se manifiestan en sus negativas, reinterpretaciones o aceptaciones parciales de los temas dominantes. (p. 24).

De esta forma, es importante el reconocimiento de los actores sociales no como receptores pasivos, sino como sujetos activos que constantemente construyen sentidos a partir de los elementos que le aporta su realidad. De toda la multiplicidad de insumos socioculturales, niños y niñas organizan de manera particular sus perspectivas frente a la sexualidad, con significaciones subjetivas propias.

Es por ello por lo que el acompañamiento educativo sobre el tema de la sexualidad debe convertirse en un imperativo actual. Asumir este compromiso favorecería el necesario avance hacia la transformación de prácticas culturales fuertemente arraigadas, que profundizan las desigualdades y la intolerancia. Es apuntarle al favorecimiento del desarroIlo integral y pleno los niños y niñas, desde una perspectiva de derechos, desde su reconocimiento como sujeto activo, partícipe, crítico, reflexivo y con capacidades para valorar las formas diversas de vida, de respetar la dignidad humana y de establecer relaciones democráticas, equitativas y pacíficas.

\section{Referencias}

Aguirre, E.; Burkart, M.; Fernández, A.; Gaspari, A.; Haftel, C. (2008). La sexualidad y los niños: ensayando intervenciones. Buenos Aires: Lugar Editorial.

Antúnez, M.; Durrieu M.; Kohen, M. (2011). Hablar de sexualidad en la escuela me da placer: experiencias de implementación de la Ley de
Educación Sexual Integral en el municipio de Tigre. Conferencia presentada en el IV Coloquio Internacional Interdisciplinario: Educación, Sexualidades y Relaciones de Género, Investigaciones, Experiencias y Relatos, Buenos Aires, Argentina.

Ayala, G. (2007). Medios de comunicación, publicidad e industria cultural: hacia la genitalización de lo erótico-sexual. Revista el Hombre y la Máquina, 28, 22-29. Recuperado de: http:// www.redalyc.org/pdf/478/47802803.pdf

Bachs, J. (1984). Conocimientos sexuales en niños/ as de 5 a 7 años. Bases para el estudio de los factores de integración. Cuadernos de Psicología, 8(2), 139-154. Recuperado de: http://www. raco.cat/index.php/QuadernsPsicologia/article/ view/10.5565-rev-psicologia.554/262712

Badinter, E. (1981). ¿Existe el amor maternal? Barcelona: Paidós-Pomaire.

Ballester, R.; Gil, M. (2006). La sexualidad en niños de 9 a 14 años. Psicothema, 18(1), 25-30. Recuperado de: http://www.psicothema.com/ psicothema. asp? $\mathrm{id}=3171$

Baquero, R.; Diker, G.; Frigerio, G. (2007). Las formas de lo escolar. Buenos Aires: Del Estante.

Barberis, E.; Taborda, D.; Zamanillo, A. (2011). Procesos de implementación del Programa Nacional de Educación Sexual Integral en la Ciudad de Córdoba. Análisis de las resignificaciones institucionales del Programa desde un estudio de casos. Conferencia presentada en el IV Coloquio Internacional Interdisciplinario: Educación, Sexualidades y Relaciones de Género, Investigaciones, Experiencias y Relatos, Buenos Aires, Argentina.

Belgich, H. E. (2004). Sujetos con capacidades diferentes: sexualidad y subjetivación. Armenia: Kinesis.

Bernal, M. (2006). Género, etnia y clase en el cine argentino. Un análisis de las representaciones de la niñez en las películas de los noventa. En S. Carli (comp.), La cuestión de la infancia. Entre la escuela, la calle y el shopping (pp. 265294). Buenos Aires: Paidós.

Bonilla, C. B. (2010). Justificaciones morales de los niños y niñas acerca de la sexualidad. Revista Latinoamericana de Ciencias Sociales, 
Niñez y Juventud, 8(2), 1013-1023. Recuperado de: http://revistaumanizales.cinde.org. co/index.php/Revista-Latinoamericana/article/ view/87/45

Boy, N. (2011). La escuela como educadora de las sexualidades. En políticas y debates sobre sexualidades, género y educación. Conferencia presentada en el IV Coloquio Internacional Interdisciplinario: Educación, Sexualidades y Relaciones de Género, Investigaciones, Experiencias y Relatos, Buenos Aires, Argentina.

Butler, J. (2000). Variaciones sobre sexo y género. En M. Lamas (comp.), El género. La construcción cultural de la diferencia sexual (pp. 303-326). México D.F.: Universidad Nacional Autónoma de México.

Camacho, L. B. (octubre, 2015). Abordaje metodológico de la sexualidad desde la perspectiva infantil. Trabajo presentado en el IV Congreso Internacional de Psicología y Educación, Psychology Investigation, Ibagué, Colombia.

Camacho, L. B.; Trujillo, M. A. (mayo, 2009). Representaciones sociales sobre niñez, adultez y sexualidad. Trabajo presentado en la XI Reunión Nacional y IV Encuentro Internacional de Investigadores sobre Juventud, Centro de Estudios sobre la Juventud, La Habana, Cuba.

Carli, S.M. (18 de septiembre de 2011). Hoy, los chicos son vistos más como consumidores que como escolares. Clarín. Recuperado de: http:// www.clarin.com/zona/Hoy-chicos-vistos-consumidores-escolares_0_556744431.html

Casati, N. (2008). Diversidad sexual. En H. Belgich, Subjetividad y violencia urbana (clase, género y racismo) (pp. 167-180). Rosario: Labor de libros.

Castoriadis, C. (1975). La institución imaginaria de la sociedad. Buenos Aires: Tusquets.

Citeli, M. T. (2005). A pesquisa sobre sexualidade e dereitos sexuals no Brasil 1990-2002: revisão crítica. Rio de Janeiro: Centro Latinoamericano em Sexualidade e Dereitos Humanos.

Congreso de la Nación Argentina. (21 de julio de 2010). Ley 26.618 y Decreto 1054/10. Ley de Matrimonio Igualitario.

. (23 de mayo de 2012). Ley 26.743 de Identidad de Género.
Conway, J. K.; Bourque, S. C.; Scott, J. W. (2000). El concepto de género. En M. Lamas (comp.), El género. La construcción cultural de la diferencia sexual (pp. 21-33). México D.F.: Universidad Nacional Autónoma de México.

Cucchiari, S. (2000). La revolución del género y la transición de la horda bisexual a la banda patrilocal: los orígenes de la jerarquía de género. En Lamas, M. (comp.), El género. La construcción cultural de la diferencia sexual (pp. 181-264). México D.F.: Universidad Nacional Autónoma de México.

Curia, M. (2006). Pequeños consumidores: algunas reflexiones sobre la oferta cultural y la construcción de identidades infantiles. En S. Carli (comp.), La cuestión de la infancia. Entre la escuela, la calle y el shopping (pp. 295-319). Buenos Aires: Paidós.

Del Río Fortuna, C. A.; Lavigne, L. (2010). Una mirada antropológica de dos políticas públicas en sexualidad en la ciudad de Buenos Aires. Propuesta Educativa, 33(1), 95-104.

Delval, J. (2001). Descubrir el pensamiento de los niños. Barcelona: Paidós.

Di Leo, P. F. (2009). Tensiones en torno a la educación sexual en escuelas medias: reflexiones desde el campo de la promoción de la salud. Argumentos. Revista de Crítica Social, 11, 83109. Recuperado de: http://publicaciones.sociales.uba.ar/index.php/argumentos/article/ view/799/685

Durán, M.; Rojas, S. (1996). La sexualidad en los niños y los adolescentes: hacia un estado del conocimiento. Santiago de Cali: Ministerio de Educación Nacional.

Foucault, M. (1974/2007). Los anormales (4ª reimpresión). Buenos Aires: Fondo de Cultura Económica.

- (1976/2008). La historia de la sexualidad: la voluntad del saber ( $3^{\text {a }}$ reimpresión). Buenos Aires: Siglo XXı.

. (1977/2006). Territorio, seguridad, población (1 ${ }^{\text {a }}$ reimpresión). Buenos Aires: Fondo de Cultura Económica.

Freud, S. (1905/1993). Obras completas. Tres ensayos para una teoría sexual (5 $5^{\mathrm{a}}$ reimpresión). Buenos Aires: Amorrortu. 
. (1907/1996). Obras completas. La ilustración sexual del niño. Madrid: Biblioteca Nueva. - (1908/1996). Obras completas. Sobre las teorías sexuales infantiles. Madrid: Biblioteca Nueva.

Gogna, M. (2005). Estado del arte. Investigación sobre sexualidad y derechos en la Argentina: 1990-2002. Buenos Aires: Cedes.

González, A.; Castellanos, B. (1996). Sexualidad y géneros: una reconceptualización educativa en los umbrales del tercer milenio. Bogotá: Magisterio.

González, L. (2000). Investigación cualitativa en psicología. Rumbos y desafíos. México D.F.: Thompson.

Hall, S. (1997). Introducción: ¿quién necesita identidad? En S. Hall.; P. du Gay (comps.), Cuestiones de identidad cultural (pp. 13-38). Buenos Aires: Amorrortu.

Hernández, R.; Fernández, C.; Baptista, P. (2007). Metodología de la investigación. México D.F.: McGraw Hill.

Katchadourian, H. (2000). La sexualidad humana: un estudio comparativo de su evolución. México D.F.: Fondo de Cultura Económica.

Lamas, M. (2000). Usos, dificultades y posibilidades de la categoría "género". En, El género. La construcción cultural de la diferencia sexual (pp. 327-366). México D.F.: Universidad Nacional Autónoma de México.

Lifschitz, M.; Pavicich, P.; Alonso, L.; Alonso, M. (2008). Sexismo en el lenguaje. Rosario: Municipalidad de Rosario.

Marina, J. A. (2002). El rompecabezas de la sexualidad. Barcelona: Anagrama.

Martín-Barbero, J. (2002). La educación desde la comunicación. Bogotá: Norma.

Martínez, M. (2006). La investigación cualitativa (síntesis conceptual). Revista de Investigación en Psicología, 9(1), 123-146. Recuperado de: http://revistasinvestigacion.unmsm.edu.pe/index.php/psico/article/view/4033/3213 DOI: https://doi.org/10.15381/rinvp.v9i1.4033

Ministerio de Educación de la Nación. (2010). Educación sexual integral para la educación inicial: contenidos y propuestas para las salas. Buenos Aires: Ministerio de Educación de la Nación.
Ministerio de Educación del Gobierno de la ciudad de Buenos Aires. Resolución núm. 3.906/SED/04.

Ministerio de Educación Nacional; Fondo de Población de las Naciones Unidas. (2008). Programa de Educación para la Sexualidad y Construcción de Ciudadanía. La dimensión de la sexualidad en la educación de nuestros niños, niñas, adolescentes y jóvenes. Bogotá: Ministerio de Educación Nacional y Fondo de Población de las Naciones Unidas.

Minzi, V. (2006). Los chicos según la publicidad. Representaciones de infancia en el discurso del mercado de productos para niños. En S. Carli (comp.), La cuestión de la infancia. Entre la escuela, la calle y el shopping (pp. 209-239). Buenos Aires: Paidós.

Ortiz, R. (1997). Modernidad-mundo e identidades. Estudios sobre las culturas contemporáneas, 3(5), 97-108. Recuperado de: http://www.culturascontemporaneas.com/contenidos/modernidad mundo.pdf

Ortiz, R. (2004). Mundialización y cultura. Bogotá: Convenio Andrés Bello.

Piaget, J. (1976). El lenguaje y el pensamiento del niño. Estudio sobre la lógica del niño (4a ed.). Buenos Aires: Guadalupe.

Quintana, A. (2006). Metodología de investigación científica cualitativa. Lima: UNMSN.

Ramos, G. A. (2009). Discurso/s que construyen sexualidades en escuelas medias de la Ciudad de Buenos Aires. En A. Villa (comp.), Sexualidad, relaciones de género y de generación. Perspectivas histórico-culturales en educación (pp. 139-169). Buenos Aires: Noveduc libros.

Sandoval, C. A. (2002). Investigación cualitativa. Bogotá: Arfo.

Steinberg, S. R.; Kincheloe, J. L. (1997). Cultura infantil, y multinacionales. La construcción de la identidad en la infancia. Madrid: Morata.

Strauss, A.; Corbin, J. (2002). Bases de la investigación cualitativa. Técnicas y procedimientos para desarrollar teoría fundamentada. MedeIlín: Universidad de Antioquia.

Sverdlik, M. (1996). La elaboración de teorías sexuales infantiles en niños de 2 a 5 años (tesis de pregrado inédita). Facultad de Psicología, Universidad de Buenos Aires, Argentina. 
Tenti, E. (2007). La escuela y la cuestión social. Ensayos de sociología de la educación. Buenos Aires: Siglo xxı.

Tomasini, M. E. (2010). Escuela y construcción de identidades de género: una aproximación a la masculinización de los varones en edad pre-escolar. Revista de Psicología, 19(1), 9-34. Recuperado de: http://200.89.78.45/index.php/RDP/ article/view/17096/17827

Urresti, M. (2008). Nuevos procesos culturales, subjetividades adolescentes emergentes y experiencia escolar. En E. Tenti (comp.), Nuevos temas en la agenda de política educativa (pp. 101-124). Buenos Aires: Siglo xxı.

Valdés, T.; Guajardo, G. (2007). Estado del arte: investigación sobre sexualidad y derechos sexuales en Chile (1990-2002). Río de Janeiro: Centro Latino Americano de Sexualidad y Derechos Humanos.

Vanegas, J. A.; Bonilla, C. B.; Camacho, L. B. (2010). Teorías sexuales infantiles en niños y niñas. Informe de investigación, Universidad Surcolombiana, Neiva, Colombia.

Werner, E. (2008). Homofobia y convivencia en la escuela. Bogotá: Universidad Pedagógica Nacional.

Zanbrini, L. (2008). Cuerpos, indumentarias y expresiones de género: el caso de las travestis de la ciudad de Buenos Aires. En M. Pecheny.; C. Figari; D. Jones (comp.), Todo sexo es político. Estudios sobre sexualidades en Argentina (pp. 123-167). Buenos Aires: Libros del Zorzal. 